\title{
Comparative IR and X-ray studies of natural and model amyloid peptides at the air/water interface
}

\author{
M. Lepère ${ }^{a}$, A.H. Muenter ${ }^{b}$, C. Chevallard ${ }^{a}$, P. Guenoun ${ }^{a}$, G. Brezesinski ${ }^{b}$,* \\ ${ }^{a}$ LIONS, Service de Chimie Moléculaire C.E.A. Saclay, 91191 Gif-sur-Yvette Cedex, France \\ ${ }^{\mathrm{b}}$ Max Planck Institute of Colloids and Interfaces, Research Campus Golm, Am Mühlenberg 1, 14476 Potsdam, Germany
}

Received 20 November 2006; received in revised form 17 January 2007; accepted 23 January 2007

Available online 30 January 2007

\begin{abstract}
Monolayers of an amyloid $\beta$ peptide $(\mathrm{A} \beta 40)$ and a much smaller model peptide (LSFD) at the air/water interface have been investigated by isotherm, IRRAS and GIXD measurements. Additionally, the LSFD monolayer has been transferred onto solid support and investigated by ATR-FTIR to test the influence of the transfer on the secondary structure of the peptide. Both peptides are surface active and form stable films of ordered $\beta$-sheet domains on the surface. The same absorption bands characteristic of an anti-parallel $\beta$-sheet conformation can be seen in the transferred LSFD film indicating that the transfer does not change the secondary structure. On the water surface, the $\beta$-sheets are oriented mostly parallel to the surface. GIXD experiments show a Bragg peak at characteristic repeat distances of $4.75-4.8 \AA$ for both peptides. The full-width at half maximum (fwhm) of this peak shows that the smaller LSFD peptide forms a monolayer film with high degree of order perpendicular to the $\beta$-strands, whereas $A \beta 40$ exhibits a drastically reduced crystallinity.
\end{abstract}

(C) 2007 Published by Elsevier B.V.

Keywords: Peptides; $\beta$-Sheet structure; Monolayers; IRRAS; GIXD; ATR-FTIR

\section{Introduction}

Several neurodegenerative diseases, such as Alzheimer's disease, are characterized by the presence of amyloid plaques in the brain. In Alzheimer's disease, the major components of these plaques are amyloid $\beta$ peptides $(\mathrm{A} \beta)$, small peptides with 39-43 amino acids. The most common constituents of the plaques are the peptides with 40 and 42 amino acids [1]. $A \beta$ peptides are the products of proteolytical cleavage of the membrane-anchored protein, amyloid precursor protein (APP) [2]. A $\beta$ peptides include the transmembrane and extracellular parts of APP, and therefore are amphipathic and surface active.

The secondary structure adopted in solution by these peptides in their native and aggregated forms are very different. In the native form, the peptides adopt usually $\alpha$-helical or unordered structures, whereas they are rich in $\beta$-sheet structures in the aggregated state [3]. In $\alpha$-helices, the hydrogen

\footnotetext{
* Corresponding author. Tel.: +49 331567 9234; fax: +49 3315679202.

E-mail address: gerald.brezesinski@mpikg.mpg.de (G. Brezesinski).
}

bonds that stabilize the structure are between groups within the same strand, whereas in $\beta$-sheets the bonds are between groups of different strands. Because the interacting $\beta$-strands are either from different regions of the same peptide or from different peptide molecules, $\beta$-sheet formation depends on the local peptide concentration and is stabilized by aggregation of the peptide molecules. Although a direct relationship between peptide aggregation and the development of Alzheimer's disease is not conclusively proved, it has been observed that the toxicity of the $A \beta$ peptide is related to the fibrillar or oligomeric forms of the peptide, but not to the monomeric form [4].

The intrinsic surface activity of $A \beta$ peptides has led to the suspected role of the cell membrane surface in the peptide aggregation pathway because the surface can serve as a point for peptide nucleation $[5,6]$. Numerous studies have probed the secondary structure behavior of different $A \beta$ peptides at natural and synthetic surfaces [7-16]. A $\beta 40$ adsorbs at hydrophobic and charged hydrophilic surfaces. At uncharged hydrophilic surfaces, no adsorption was observed. Hydrophobic surfaces induce rapid $\beta$-sheet formation $[7,8]$. Fluorinated nanoparticles made of a polyampholyte-fluorosurfactant complex induce $\alpha$-helix rich 


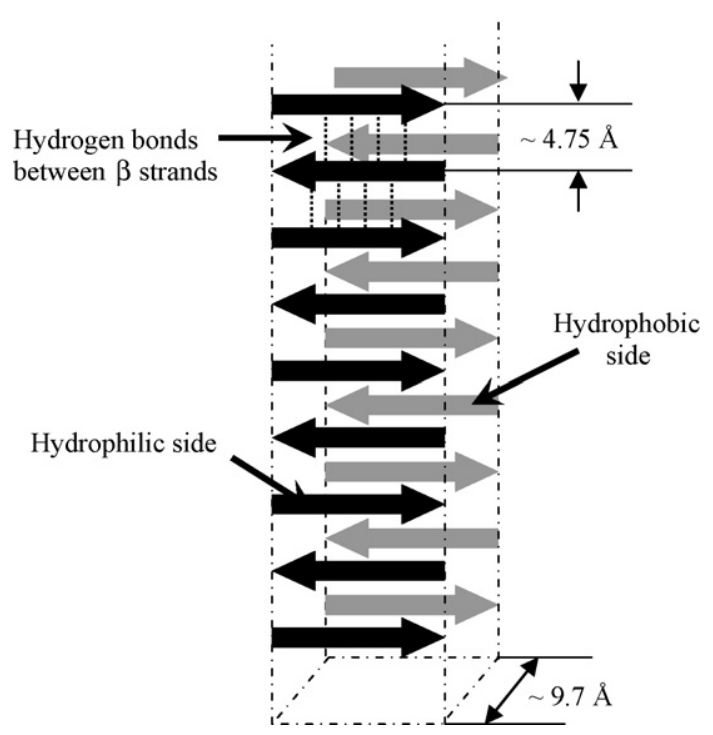

Fig. 1. Cross- $\beta$ structure of amyloid fibrils: amphiphilic $\beta$-strand filaments result from intermolecular hydrogen bonding. The filaments associate in pairs to bury their hydrophobic sides. Higher scale organization of the filaments leads to amyloid fibril formation.

structures in $\mathrm{A} \beta 40$, whereas their hydrogenated analogues lead to $\beta$-sheet formation [7].

It is instructive to compare the surface behavior of $A \beta$ peptides with other peptide sequences. $A \beta$ peptides are both difficult to handle and too large for atomistic simulation studies. Additionally, studies of model peptides provide insight into more universal, non-sequence dependent, behavior of $\beta$-strand forming peptides at surfaces. Therefore, we present here studies of a much smaller model peptide, which also forms amyloidlike fibrils, and compare its $\beta$-sheet structures formed at the air-water interface to the $\beta$-sheet structures formed by an $A \beta$ peptide.

Our model peptide is a 12-amino acid peptide that we will refer to as LSFD. It derives its primary sequence from the cell attachment fiber protein of the human adenovirus Ad2 [17]. While the native trimeric protein forms a triple $\beta$-spiral in solution [18], the LSFD adopts a $\beta$-sheet conformation in bulk and progressively aggregates into amyloid-like fibrils. These fibrils form in a "cross- $\beta$ structure", which corresponds to an elongated $\beta$-sheet structure, with the long axis running perpendicular to the $\beta$-strands, and develops through extensive intermolecular hydrogen bonding (see Fig. 1). Therefore, A $\beta 40$ and LSFD peptides are both produced by cleavage of a larger protein and subsequently adopt a new secondary structure. However, since the LSFD primary sequence is very different from that of $A \beta 40$, a comparison of these two peptides provides sequence independent information on amyloid behavior.

Monolayers of the LSFD have been prepared at the air-water interface and the peptide's secondary structure and packing order as a function of surface concentration has been studied. We compare the results of infrared and X-ray diffraction experiments on the LSFD monolayer to monolayers of $A \beta(1-40)$ also formed at the air-water interface.

\section{Experimental}

\subsection{Materials}

Aß40 (DAEFRHDSGYEVHHQKLVFFAEDVGSNKGAIIGLMVGGVV) was obtained from Bachem (Switzerland). The peptide was first dissolved in hexafluoroisopropanol (HFIP) to destroy possible aggregates and stored in a refrigerator. Before measurements, HFIP was evaporated under a nitrogen stream and the peptide was dissolved in water. The secondary structure of the peptide was checked using circular dichroism (CD) spectroscopy (Jasco J-715, Japan). The peptide was found to have predominantly random coil conformation after such a pretreatment.

Lyophilized LSFD (LSFDNSGAITIG-NH ${ }_{2}$ ) peptide samples were kindly provided by Dr. J.-F. Hernandez (CNRS, UMR5810 Faculté de Pharmacie, Montpellier) in the form of a trifluoroacetate salt. The $\mathrm{C}$-terminal amide peptide was manually synthesised by the stepwise solid-phase method [17]. Synthesis was performed on a 4-methyl benzhydrylamine resin, following standard Boc (tert-butyl-oxy-carbonyl) chemistry and HBTU ( $O$-benzotriazole- $N, N, N^{\prime}, N^{\prime}$-tetramethyl-uroniumhexafluoro-phosphate) as the coupling agent. Cleavage of the peptide from the resin and simultaneous deprotection of side chains were carried out using fluorhydric acid in the presence of anisole as scavenger. The peptide was purified by reversephase HPLC. It exhibits 95\% purity, as assessed by reverse-phase HPLC, and its identity was confirmed by electro-spray mass spectrometry. The molecular mass, including one trifluoroacetate molecule per one LSFD peptide, is $1307.3 \mathrm{~g} / \mathrm{mol}$.

\subsection{Methods}

IRRAS (infrared reflection absorption spectroscopy) and GIXD (grazing incidence X-ray diffraction) experiments were carried out both on A $\beta 40$ and LSFD monolayers at the air/water interface, allowing in situ characterization of the peptide conformation. The subphase was Milli-Q deionized water (resistivity of $18.2 \mathrm{M} \Omega \mathrm{cm}$ ). The surface tension was recorded with a continuous Wilhelmy-type pressure measuring system using a filter paper as plate. By means of movable barriers the peptide monolayer was compressed and surface pressure/area isotherms were recorded continuously at a given temperature. The vertical Langmuir-Blodgett deposition of the LSFD peptidic film on quartz substrates enabled ATR-FTIR (attenuated total reflection Fourier transform infrared spectroscopy) measurements.

\subsubsection{Monolayer formation}

The peptide surface films were formed on a pure water subphase contained in a Teflon Langmuir trough. All experiments were performed at $20^{\circ} \mathrm{C}$. The solubility but high surface activity of $A \beta 40$ in water allows the formation of two types of $A \beta 40$ monolayers. Although $A \beta 40$ is water soluble, when it is trapped at the air/water interface the desorption kinetics are very slow. The peptide solution can be either directly spread at the water surface to form a Langmuir monolayer or injected into the water 
subphase so that a slow adsorption process leads to the formation of a Gibbs monolayer at the air-water interface.

The adsorption method could not be employed with the LSFD peptide because of its strong propensity to form insoluble fibrils in water, which could prevent molecular adsorption. However, the lyophilized peptide could be easily dissolved in trifluoroacetic acid (TFA, Fluka). This solvent is known to break down aggregated $\beta$-sheet structures and most likely induces a disordered conformation of the LSFD peptide [3]. Peptide solutions were further diluted by addition of chloroform $\left(\mathrm{CHCl}_{3}\right.$, Baker $)$ to facilitate spreading of the solution at the interface. The final concentration of the LSFD solution was $0.07 \mathrm{mg} / \mathrm{mL}$ with a 1 (TFA):9 $\left(\mathrm{CHCl}_{3}\right)$ solvent ratio.

\subsubsection{IRRAS}

2.2.2.1. IRRAS measurements. In the IRRAS measurements, the IR beam was conducted out of the IFS 66 FTIR spectrometer (Bruker, Germany), polarized and directed to the liquid surface by an external reflection unit (XA-511, Bruker). A mercury-cadmium-telluride (MCT) detector collected the reflected signal. An enclosed, two-reservoir Teflon trough system was used in each measurement to minimize the effect of water vapor in the spectra. The trough was shuttled so that in each measurement the IR beam was incident first on the bare water surface (reference) and then on the monolayer covered surface (sample). The infrared measurements are reported in reflectance-absorbance (RA) units where $\mathrm{RA}=-\log [$ (sample reflectivity) $/($ reference reflectivity $)]=-\log \left(R / R_{0}\right)$. The resolution used in all experiments was $8 \mathrm{~cm}^{-1}$. The scanner velocity was set to $20 \mathrm{kHz}$. Spectra were co-added over 400 scans for p-polarized and over 200 scans for s-polarized light.

2.2.2.2. Spectra simulations. The formulism used to simulate the experimental spectra is based on that presented most recently by Mendelsohn and coworkers [19,20]. Briefly, the calculation uses the method of Kuzmin and Michailov to calculate the reflection coefficients of the three layered (air, peptide monolayer, and water) system. The calculated optical constants in this treatment depend on the orientation of the studied vibration. The simulated spectra in Fig. 3b assume that the system has uniaxial symmetry or that the $\beta$-sheets have a random orientation in the $x-y$ plane. The shapes of the simulated spectra are thus determined by the orientation of the $\beta$-sheet vertically with respect to the surface plane. The spectra in Fig. $3 \mathrm{~b}$ are modeled for a $\beta$-sheet that lies exactly flat in the surface plane. The oscillator strength, $M$, for each vibration $\left(M_{\text {amide I }}=1.6 M_{\text {amide II }}\right)$ and film thickness $(9 \AA)$ are chosen to most closely resemble the experimental spectra.

\subsubsection{ATR-FTIR}

FTIR spectra of transferred LSFD films were recorded in the attenuated total reflection mode using a Bruker spectrometer (Vertex $70^{\circledR}$ ) equipped with a single reflection ATR unit (Miracle ${ }^{\circledR}$ unit) and an MCT detector. The LSFD film, once deposited on a perfectly flat quartz wafer, was pressed in contact with the diamond plate of the ATR unit. Each spectrum was derived from 1024 scans performed at a $2 \mathrm{~cm}^{-1}$ frequency res- olution. A blank quartz wafer was used as background in all measurements.

\subsubsection{GIXD}

Grazing incidence X-ray diffraction measurements on the peptide monolayers were carried out at HASYLAB (Hamburg, Germany) using the BW1 beam line of the DESY synchrotron source [21-23]. The peptide solution was either adsorbed $(\mathrm{A} \beta 40)$ or spread (LSFD) at the air/water interface. The monochromatic X-ray beam $(\lambda=1.304 \AA)$ was adjusted to strike the air-water interface at the grazing incidence angle $\alpha_{\mathrm{i}}=0.85 \alpha_{\mathrm{c}}$, where $\alpha_{\mathrm{c}}$ is the critical angle for total reflection of the X-ray beam on the water surface. The dimensions of the Xray beam footprint on the interface were about $2 \mathrm{~mm} \times 50 \mathrm{~mm}$. GIXD diffraction signals result from ordered domains at the air-water interface. The scattered intensity was detected by a linear position-sensitive detector (PSD, OEM-100-M, Braun, Garching, Germany). The PSD detector was gradually rotated to scan the in-plane $q_{x y}$ component values of the scattering vector. The out-of-plane $q_{z}$ component of the scattering vector was detected in the range $0.0 \AA^{-1} \leq q_{z} \leq 1 \AA^{-1}$. The diffraction data presented below are Bragg peaks obtained by integrating the diffracted intensity over a specified $q_{z}$ window of the PSD. The $q_{x y}$ positions of the Bragg peaks yield the in-plane lattice repeat distances $d=2 \pi / q_{x y}$ of the interfacial crystallites. The in-plane coherence length $L_{x y}$, a measure of the range of the crystalline order, can be inferred from the full-width at half maximum (fwhm) of the Bragg peaks according to $L_{x y} \sim 0.9(2 \pi) /$ fwhm $\left(q_{x y}\right)$.

\section{Results and discussion}

\subsection{LSFD Isotherm}

The interfacial peptidic film was built by spreading $200 \mu \mathrm{l}$ of the LSFD solution on the ultrapure water ( $\mathrm{pH}$ 5.8) subphase. The TFA solvent dissolved right away into the subphase, reducing the $\mathrm{pH}$ to 2.6. The monolayer was allowed to relax for $30 \mathrm{~min}$ before it was compressed at a constant rate of $7.44 \AA^{2} /($ molecule min). Fig. 2 shows the isotherm recorded during such a compres-

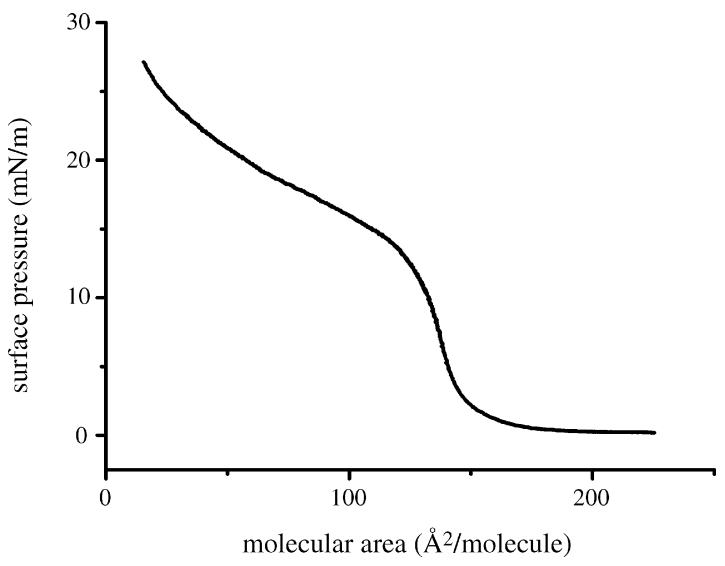

Fig. 2. Langmuir isotherm of the LSFD monolayer (compression rate: $7.44 \AA^{2} /($ molecule min $)$. 


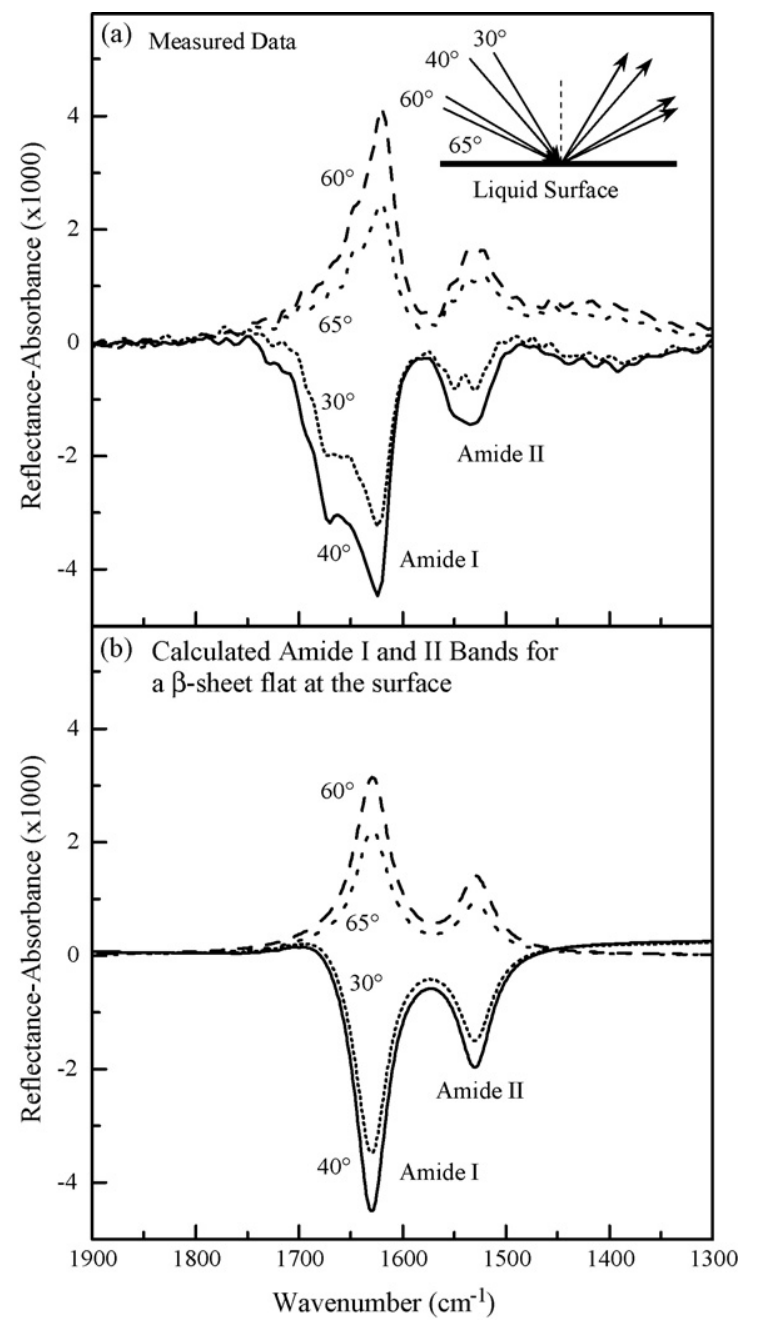

Fig. 3. (a) Angle dependent experimental p-polarized IRRA spectra of a LSFD monolayer spread on water at $20^{\circ} \mathrm{C}$. The monolayer was compressed to $3 \mathrm{mN} / \mathrm{m}$ and allowed to relax to $2 \mathrm{mN} / \mathrm{m}$ before the measurement. (b) Simulated ppolarized IRRA spectra of a LSFD monolayer under the assumption that the peptide lies flat at the interface. Simulation parameters: tilt angle $(\theta)=$ twist angle $(\chi)=90^{\circ}, v_{\text {amide I }}=1630 \mathrm{~cm}^{-1}, v_{\text {amide II }}=1530 \mathrm{~cm}^{-1} ; M_{\text {amide I }}=1.6 M_{\text {amide II }}$; fwhm: $40 \mathrm{~cm}^{-1}$, monolayer thickness: $9 \AA$; monolayer refractive index: 1.4 ; polarizer efficiency: $98.2 \%$.

sion. The surface activity of the LSFD peptide manifests itself in a raise of the surface pressure at a molecular area below $150 \AA^{2} /$ molecule. This value closely matches the molecular area of a $\beta$-sheet molecule lying flat on the water surface, suggesting the existence of a dense $\beta$-sheet monolayer at the air-water interface.

\subsection{IRRAS}

Infrared measurements, using IRRAS, reveal anti-parallel $\beta$ sheet structure in the LSFD monolayer at all surface pressures along the isotherm. The spectra in the top panel of Fig. 3a were taken after the LSFD peptide film was compressed to $3 \mathrm{mN} / \mathrm{m}$ and allowed to relax at a constant surface area to $2 \mathrm{mN} / \mathrm{m}$. The Amide I band at $1624 \mathrm{~cm}^{-1}$ is diagnostic of $\beta$-sheet structure. The broad Amide II band centered at $1540 \mathrm{~cm}^{-1}$ is also consistent with the $\beta$-sheet structure [24]. The band at $1670 \mathrm{~cm}^{-1}$ arises from residual TFA from both the spreading solution and the peptide synthesis. The $1690 \mathrm{~cm}^{-1}$ peak that is diagnostic of anti-parallel $\beta$-sheet structure is seen as a shoulder on the TFA band. A more detailed analysis of spectra from this experiment combined with further LSFD monolayer experiments confirms the presence of anti-parallel $\beta$-sheet structure in the LSFD monolayer.

In previously studied $\beta$-sheet forming peptide monolayers, the $\beta$-sheet structure forms with the sheet plane mainly parallel to the surface $[8,20,24-27]$. We confirmed that the $\beta$-sheet structures in the LSFD monolayer lie mainly in the surface plane by collecting p-polarized spectra at several IR beam angles. For a vibration that corresponds to a dipole oscillating primarily in the surface plane, the reflectivity from the sample surface will be greater than the reflectivity from the reference surface $\left(R>R_{0}\right)$ for a p-polarized IR beam incident at angles smaller than the Brewster angle $\left(\sim 53^{\circ}\right)$. The reverse $\left(R<R_{0}\right)$ is true for incident angles larger than the Brewster angle. Therefore, the direction of the peaks in a reflectance-absorbance $\left(\mathrm{RA}=-\log \left(R / R_{0}\right)\right)$ spectrum will be opposite for spectra collected above and below the Brewster angle. As shown in the top panel of Fig. 3, the measured LSFD spectra display this characteristic behavior for an infrared vibration with a transition dipole moment primarily parallel to the surface. The transition dipole moments for both the amide vibrations are in the plane of the $\beta$-sheet. The spectrum therefore must result from $\beta$-sheet structures that lie mainly in the surface plane.

Fig. $3 \mathrm{~b}$ shows calculated reflectance-absorbance spectra of the Amide I and II bands of an anti-parallel $\beta$-sheet and is meant for qualitative comparison with the experimental spectra in the upper panel. A comparison shows that the relative height and direction of each peak at the different angles are the same for both the measured and simulated spectra. Therefore, the LSFD $\beta$-sheet lies mainly flat in the surface plane. Discrepancies in relative band height between the simulated and experimental spectra likely arise either from a slight tilt or twist out of the surface plane for LSFD $\beta$-sheets or a system that has a degree of uniaxial asymmetry. A further publication will address a more quantitative simulation of the LSFD spectra and describe a more specific $\beta$-sheet orientation.

A $\beta 40$ as a water soluble peptide can be studied by injecting the amyloid peptide into the subphase and observing the adsorption at the interface. Under such conditions, $A \beta 40$ forms a stable $\beta$-sheet network at the water surface [8]. However, $A \beta 40$ monolayer can also be prepared by directly spreading the pretreated peptide at the interface. Fidelio and coworkers used a three-component (chloroform, methanol, and DMSO) spreading solutions to create peptide monolayers of amyloid $\beta(1-42)$ [28]. Experiments in our laboratory have shown that spreading the $\mathrm{A} \beta 40$ from a dilute peptide $(0.5 \mathrm{mg} / \mathrm{mL})$ and $0.1 \mathrm{M}$ ammonium hydroxide solution resulted in monolayers similar to those Aß40 monolayers formed using a chloroform, methanol, and DMSO spreading solvent (result not shown). Fig. 4 illustrates that the infrared spectra from an adsorbed versus a spread $A \beta 40$ (without extra spreading solvent) monolayer contain the same spectral features. This result indicates that the general structure of the $\mathrm{A} \beta 40$ monolayer is independent of the layer preparation. 


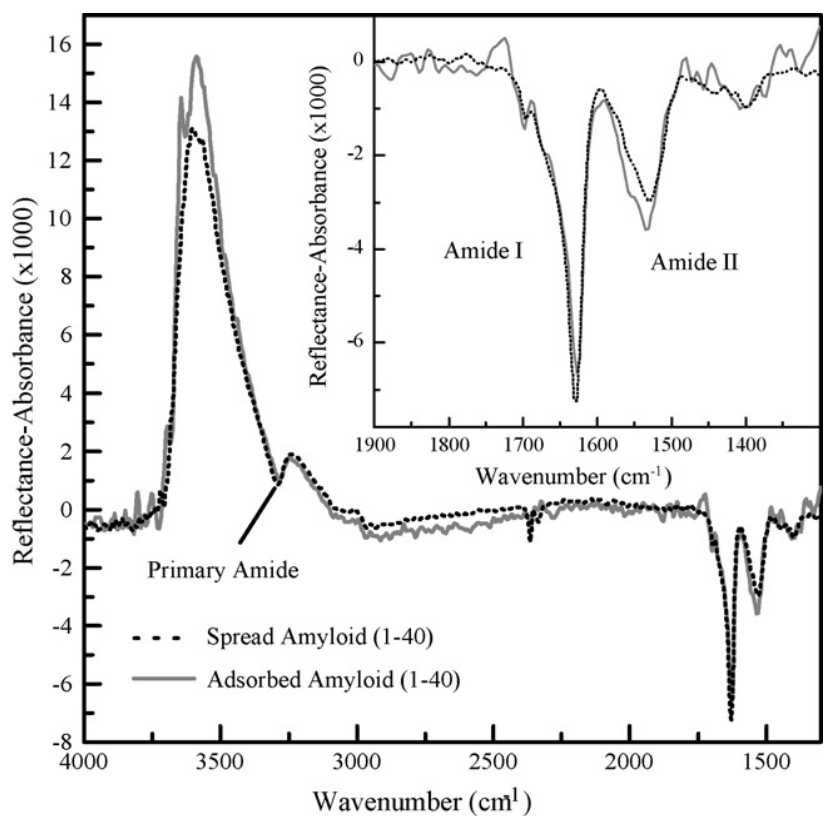

Fig. 4. Comparison of IRRA spectra for an adsorbed $(9 \mathrm{mN} / \mathrm{m})$ and spread $(20 \mathrm{mN} / \mathrm{m})$ amyloid $\beta(1-40)$ monolayer on water at $20^{\circ} \mathrm{C}$. Spectra were taken with p-polarized light at a $40^{\circ}$ incident angle.

The strong Amide I peak in the $1630 \mathrm{~cm}^{-1}$ region indicates a well-formed $\beta$-sheet structure. Although, the two spectra in Fig. 4 were taken at different surface pressures, the similar heights of the $\mathrm{OH}$ stretching peak at $3600 \mathrm{~cm}^{-1}$ indicate an equivalent thickness for both layers. The adsorbed $\mathrm{A} \beta 40$ monolayer at $9 \mathrm{mN} / \mathrm{m}$ has reached the equilibrium surface pressure for the peptide concentration of the bulk solution. The spread and then compressed $\mathrm{A} \beta 40$ monolayer remains in a metastable state. When left to relax at a constant area per molecule, the IRRAS signal remained unchanged over $12 \mathrm{~h}$, but the surface pressure dropped from 20 to $12 \mathrm{mN} / \mathrm{m}$.

\subsection{ATR-FTIR}

Technological applications involving organic films often require that the film must be transferred onto a solid substrate. Therefore, ATR-FTIR spectroscopy measurements were performed with a LSFD film deposited on a quartz wafer to check the influence of the transfer on the peptide conformation. Fig. 5 shows an ATR spectrum obtained for a film deposited at $7 \mathrm{mN} / \mathrm{m}$. Again, the absorption peaks characteristic of an anti-parallel beta-sheet conformation can be seen: amide I bands at 1624 and $1695 \mathrm{~cm}^{-1}$, as well as the amide II band at $1527 \mathrm{~cm}^{-1}$. The same results were obtained for films deposited at lower and higher surface pressures, respectively. The deposition had therefore no influence on the peptide conformation, although no information about the influence of the transfer on the molecule orientation could be obtained using this technique.

\subsection{GIXD}

GIXD experiments previously performed on $A \beta 40$ clearly show the existence of $\beta$-sheet ordered domains at the air-water

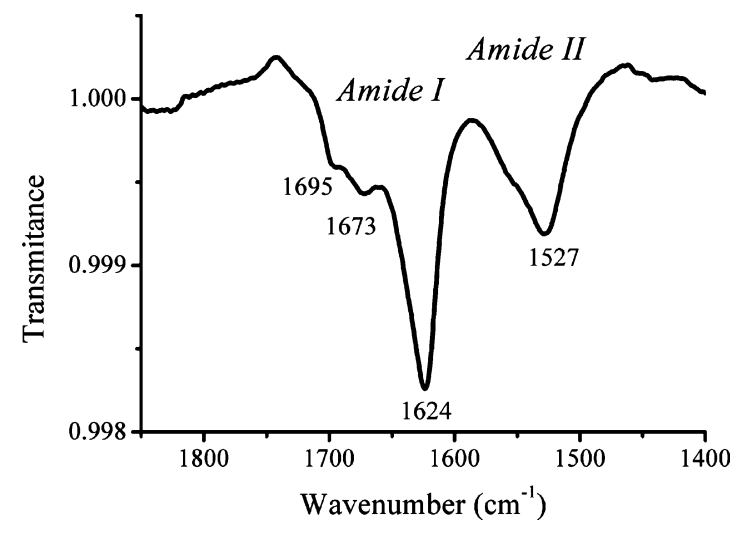

Fig. 5. FTIR-ATR spectrum of an LSFD monolayer transferred onto a quartz wafer by vertical Langmuir-Blodgett deposition at a surface pressure of $7 \mathrm{mN} / \mathrm{m}$.

interface. As reproduced in Fig. 6, the A $\beta 40$ monolayer at a surface pressure equal to $14 \mathrm{mN} / \mathrm{m}$ exhibits a pronounced Bragg peak at $q_{x y}=1.33 \AA^{-1}$ and $q_{z}=0 \AA^{-1}$. This peak is visible for all surface pressures along the adsorption isotherm, although with varying intensity. The peak position corresponds to a repeat distance of $4.75 \AA$ that is characteristic of the spacing between peptide backbones interconnected by hydrogen bonds in a $\beta$ sheet conformation. The A $\beta 40$ monolayer possesses crystalline order in the direction perpendicular to the $\beta$-strands. The coherence length $L_{x y}$ of the Bragg peak gives the range of this translational order: $L_{x y}=97 \AA$.

Similar GIXD experiments were performed on the LSFD monolayer to obtain more information on the film structure and to determine any common characteristics of the natural and model peptides. Here again, a Bragg peak corresponding to a $\beta$-sheet network was observed at all pressures along the compression curve. Fig. 6 presents such a Bragg peak at $q_{x y}=1.31 \AA^{-1}$ obtained for a LSFD monolayer relaxed to $10.1 \mathrm{mN} / \mathrm{m}$. This position corresponds to a repeat distance of

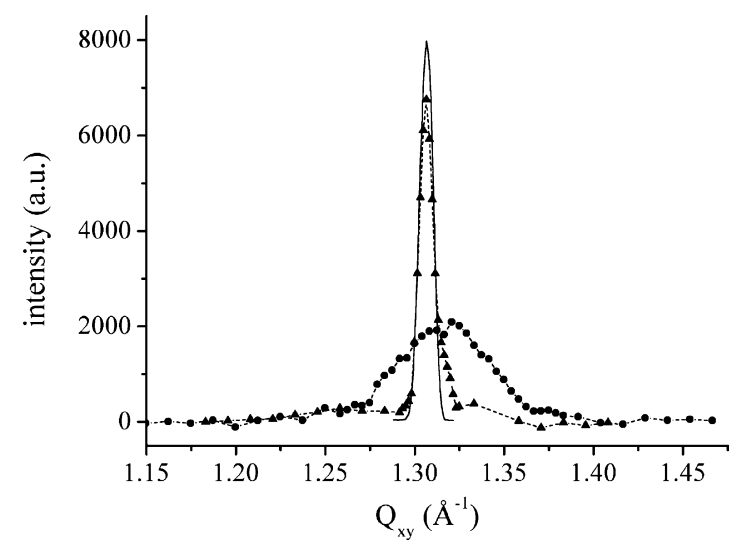

Fig. 6. Comparison of Bragg peaks for an A $\beta 40$ monolayer and a LSFD monolayer. The broad peak is the intensity diffracted by an A $\beta 40$ monolayer adsorbed at the air-water interface at $14 \mathrm{mN} / \mathrm{mn}$ (solid circles, reproduced from Ref. [8]). A much narrower curve is obtained for a LSFD monolayer compressed to a final relaxed pressure of $10.1 \mathrm{mN} / \mathrm{m}$ (solid triangles). In both cases, the diffracted intensity was integrated over a $q_{z}$ window from 0 to $0.3 \AA^{-1}$. The full-width at half maximum (fwhm) provides an estimate of the coherence length. The solid line represents the resolution function determined by the Soller collimator. 
$d=4.8 \AA$. The width of this Bragg peak is resolution limited implying a coherence length larger than $575 \AA$. For comparison, the peak arising from the reflection of the direct beam is included in Fig. 6. The fwhm of this peak gives the resolution determined by a Soller collimator in front of the PSD $\left(\Delta q_{x y}=0.0074 \AA^{-1}\right)$. The higher crystallinity of the LSFD film likely arises because every residue of this short peptide participates in the linear $\beta$-strand. In contrast, simulations and NMR data show that the $\beta$-sheet structures of $A \beta 40$ do not encompass every residue $[29,30]$. Random coil structures likely prevent a longer range order in and thus a greater crystallinity of the $A \beta 40$ film.

Analogous to other beta-sheet forming peptide monolayers, a two-dimensional ordering of the peptides at the interface was also observed by GIXD [31]. A second diffraction peak at much lower $q_{x y}$ values was seen for each peptide film and indicates a repeat distance corresponding to the end-to-end distance of each peptide strand. For $\AA \beta 340$, the end-to-end spacing is $d \sim 80 \AA$ [32] whereas $d \sim 40 \AA$ for the shorter LSFD peptide. The 2D crystalline order of the peptide films is currently under further investigation.

These results show that the LSFD peptide, chosen as a model amyloid peptide, behaves similarly but not identically to the A $\beta 40$. Like its natural counterpart, it forms a $\beta$-sheet monolayer at the air-water interface, and develops a strong crystalline order in the direction perpendicular to the $\beta$-strands. However, the much longer coherence length indicates that the crystalline order in the LSFD monolayer is much greater than in the A $\beta 40$ monolayers.

\section{Conclusions}

This initial investigation of the film properties of a monolayer of the LSFD peptide confirms that the peptide, under the conditions investigated, behaves comparable to a monolayer of the larger $\mathrm{A} \beta 40$ peptide. The mix of hydrophobic and hydrophilic residues in the LSFD and A 340 leads to their surface activity. Both peptides form stable films of ordered $\beta$-sheet domains on the surface. The plane of the sheets is parallel to the surface. The hydrogen bonds between different peptide strands almost certainly help to stabilize each film. The much smaller LSFD peptide forms a monolayer film with a high degree of order perpendicular to the $\beta$-strands. It is likely that all of the residues of the LSFD peptide participate in a single $\beta$-strand structure that can then assemble into a well-ordered domain. Unordered regions of the peptide are assumed to reduce the crystalline order of the $A \beta 40$ peptide at the surface. This reduced crystallinity is observed as a shorter coherence length for the intra-strand spacing for the $A \beta 40$ peptide monolayer as compared to the LSFD peptide monolayer. Self-assembled peptides and proteins have emerged as building blocks for constructing functional nanomaterials [33]. In particular, highly ordered peptide assemblies, transferred onto solid surfaces, such as LSFD monolayers on quartz could be used for nanometer-scale surface patterning. These patterns may form selective nucleation sites that then can be used to induce technologically relevant crystalline nanostructures.

\section{Acknowledgements}

This work has been supported by the French-German CEA/CNRS/DFG/MPG network "Thin films of complex fluids: from two to three dimensions". M.L., C.C. and P.G. also thank J.F. Hernandez and A. Mitraki for the synthesis and characterization of LSFD, and the Ministere de la Recherche for a grant through the ACI Nanosciences. We thank HASYLAB at DESY (Hamburg, Germany) for beam time and providing excellent facilities and support.

\section{References}

[1] D.J. Selkoe, Physiol. Rev. 81 (2001) 741.

[2] G.C. Gregory, G.M. Halliday, Neurotoxic. Res. 7 (2005) 29.

[3] C.J. Barrow, A. Yasuda, P.T.M. Kenny, M.G. Zagorski, J. Mol. Biol. 225 (1992) 1075.

[4] D.M. Walsh, D.J. Selkoe, Protein Peptide Lett. 11 (2004) 213.

[5] J. McLaurin, A. Chakrabartty, J. Biol. Chem. 271 (1996) 26482.

[6] K. Matsuzaki, C. Horikiri, Biochemistry 38 (1999) 4137.

[7] S. Rocha, A.F. Thünemann, M.C. Pereira, M.A.N. Coelho, H. Möhwald, G. Brezesinski, Chem. BioChem. 6 (2005) 280.

[8] E. Maltseva, A. Kerth, A. Blume, H. Möhwald, G. Brezesinski, Chem. BioChem. 6 (2005) 1817.

[9] E. Terzi, G. Holzemann, J. Seelig, Biochemistry 36 (1997) 14845.

[10] L.P. ChooSmith, W. GarzonRodriguez, C.G. Glabe, W.K. Surewicz, J. Biol. Chem. 272 (1997) 22987.

[11] F. Lindstrom, M. Bokvist, T. Sparrman, G. Grobner, Phys. Chem. Chem. Phys. 4 (2002) 5524.

[12] C.E. Giacomelli, W. Norde, Macromol. Biosci. 5 (2005) 401.

[13] W.G. Wood, G.P. Eckert, U. Igbavboa, W.E. Muller, Biochim. Biophys. Acta: Biomembr. 1610 (2003) 281.

[14] M. Bokvist, F. Lindstrom, A. Watts, G. Grobner, J. Mol. Biol. 335 (2004) 1039.

[15] G.H. Wu, J. Majewski, C. Ege, K. Kjaer, M.J. Weygand, K.Y.C. Lee, Phys. Rev. Lett. 93 (2004) 028101.

[16] A. Kakio, Y. Yano, D. Takai, Y. Kuroda, O. Matsumoto, Y. Kozutsumi, K. Matsuzaki, J. Peptide Sci. 10 (2004) 612.

[17] K. Papanikolopoulou, G. Schoehn, V. Forge, V.T. Forsyth, C. Riekel, J.F. Hernandez, R.W.H. Ruigrok, A. Mitraki, J. Biol. Chem. 280 (2005) 2481.

[18] M.J. van Raaij, A. Mitraki, G. Lavigne, S. Cusack, Nature 401 (1999) 935.

[19] J.W. Brauner, C.R. Flach, Z. Xu, X.H. Bi, R. Lewis, R.N. McElhaney, A. Gericke, R. Mendelsohn, J. Phys. Chem. B 107 (2003) 7202.

[20] Z. Xu, J.W. Brauner, C.R. Flach, R. Mendelsohn, Langmuir 20 (2004) 3730

[21] J. Als-Nielsen, D. Jacquemain, K. Kjaer, F. Leveiller, M. Lahav, L. Leiserowitz, Phys. Rep. 246 (1994) 252.

[22] R. Rietz, W. Rettig, G. Brezesinski, W.G. Bouwman, K. Kjaer, H. Möhwald, Thin Solid Films 285 (1996) 211.

[23] D. Jacquemain, F. Leveiller, S.P. Weinbach, M. Lahav, L. Leiserowitz, K. Kjaer, J. Als-Nielsen, J. Am. Chem. Soc. 113 (1991) 7684.

[24] T. Buffeteau, B. Desbat, Appl. Spectrosc. 43 (1989) 1027.

[25] S. Castano, B. Desbat, Biochim. Biophys. Acta: Biomembr. 1715 (2005) 81.

[26] P. Cai, C.R. Flach, R. Mendelsohn, Biochemistry 42 (2003) 9446.

[27] A. Kerth, A. Erbe, M. Dathe, A. Blume, Biophys. J. 86 (2004) 3750.

[28] E.E. Ambroggio, D.H. Kim, F. Separovic, C.J. Barrow, K.J. Barnham, L.A. Bagatolli, G.D. Fidelio, Biophys. J. 88 (2005) 2706.

[29] Y.C. Xu, J.J. Shen, X.M. Luo, W.L. Zhu, K.X. Chen, J.P. Ma, H.L. Jiang, Proc. Natl. Acad. Sci. U.S.A. 102 (2005) 5403.

[30] N.A. Whittemore, R. Mishra, I. Kheterpal, A.D. Williams, R. Wetzel, E.H. Serpersu, Biochemistry 44 (2005) 4434.

[31] H. Rapaport, K. Kjaer, T.R. Jensen, L. Leiserowitz, D.A. Tirrell, J. Am. Chem. Soc. 122 (2000) 12523.

[32] G. Brezesinski, E. Maltseva, H. Möhwald, Z. Phys. Chem. 221 (2007) 95.

[33] K. Rajagopal, J.P. Schneider, Curr. Opin. Struct. Biol. 14 (2004) 480. 